\title{
Response to "Metformin use and risk of COVID-19 among patients with type II diabetes mellitus: an NHIS-COVID-19 database cohort study"
}

\author{
Satesh $\operatorname{Kumar}^{1}{ }^{(D)} \cdot$ Roomi Raja $^{2}$ (I)
}

Received: 19 December 2021 / Accepted: 28 January 2022 / Published online: 4 March 2022

(c) Springer-Verlag Italia S.r.l., part of Springer Nature 2022

Dear editor,

We have read the article "Metformin use and risk of COVID-19 among patients with type II diabetes mellitus: an NHIS-COVID-19 database cohort study" by Oh and Song et al. [1]. It was auspicious to read this article, and the author's efforts are to be cherished. We agree with the final piece of information that metformin therapy did not have a significant impact on hospital mortality in COVID-19 patients. However, it would be a privilege to append a few points to enrich the study's findings.

Firstly, the single-centered database study could have drawn out various concerns for the study's validity. For instance, a 2020 multicentered study conducted in China included data from 16 different hospitals, resulting in significant findings [2]. Secondly, the author could widen exclusion criteria in their study to avoid possible bias in the study. For illustration, research excluded patients with fatal conditions such as myocardial infarction, stroke, and pulmonary embolism, pregnant patients other than younger cohorts, and patients with incomplete documentation [2]. Based on varied research, contradictory results have been proposed. A 2021 meta-analysis, pooled study data from various countries, including the USA, China, India, Italy, UK, and

Managed by Antonio Secchi .

This comment refers to the article available online at https://doi. org/10.1007/s00592-020-01666-7.

Satesh Kumar

Kewlanisatish@gmail.com

Roomi Raja

romirajagoindani@yahoo.com

1 Shaheed Mohtarma Benazir Bhutto Medical College Liyari, Karachi, Parsa citi Block E Floor 5th, Flat 501 Near Police Headquarter, Garden East Karachi, Pakistan

2 Ziauddin University, Karachi, Pakistan
France, concluded that antidiabetic drug metformin is significantly associated with a protective effect in COVID-19 patients [3, 4], as established metformin is associated with significant adverse effects such as acidosis and metallic taste. Hence, authors should have mentioned specific contraindications concerning these adverse effects as it could impact patients' health in the future with significant comorbidities. For instance, metformin was contraindicated in patients with severe respiratory, heart, and renal problems suffering from COVID-19. Likewise, a 2020 meta-analysis reported that metformin might be associated with reduced mortality in patients with COVID-19 [4]. Authors should also have reported different basic laboratory tests which could have provided a better idea of patient's health at that time. A 2020 study reported different baseline laboratory values such as complete blood count and compared it between patients of metformin and non-metformin groups. Authors should also have briefed about different comorbidities such as hypertension, chronic obstructive pulmonary diseases, and malignancies in the study group since these chronic diseases greatly impact mortality [4]. Different studies have reported numerous findings which raise a question regarding the use of metformin in a patient with diabetes. However, most studies also recommend using metformin in COVID-19 patients due to its protective role in blood glucose control [2-5]. A 2021 study also proposed that diabetes directly impacts mortality in COVID-19 patients; hence, metformin may provide a protective role in a high-risk population [5].

Finally, new studies should be conducted on antidiabetic medications such as metformin in SARS-COV patients, as more than $10.5 \%$ of the adult population suffers from diabetes.

\section{Acknowledgements None.}

Authors' contributions The authors contributed to data collection, literature review, and manuscript writing. 
Funding None.

Availability of data and materials Not applicable.

\section{Declarations}

Conflict of interest The authors declare that they have no conflict of interest to this work.

Ethics approval and consent to participate Not applicable.

Consent for publication Not applicable.

\section{References}

1. Oh TK, Song IA (2021) Metformin use and risk of COVID19 among patients with type II diabetes mellitus: an NHISCOVID-19 database cohort study. Acta Diabetol 58(6):771-778. https://doi.org/10.1007/s00592-020-01666-7
2. Cheng X, Liu YM, Li H et al (2020) Metformin is associated with higher incidence of acidosis, but not mortality, in individuals with COVID-19 and pre-existing type 2 diabetes. Cell Metab 32(4):537-547.e3. https://doi.org/10.1016/j.cmet.2020.08.013

3. Samuel SM, Varghese E, Büsselberg D (2021) Therapeutic potential of metformin in COVID-19: reasoning for its protective role. Trends Microbiol 29(10):894-907. https://doi.org/10.1016/j.tim. 2021.03.004

4. Luo P, Qiu L, Liu Y et al (2020) Metformin treatment was associated with decreased mortality in COVID-19 patients with diabetes in a retrospective analysis. Am J Trop Med Hyg 103(1):69-72. https://doi.org/10.4269/ajtmh.20-0375

5. Crouse A, Grimes T, Li P, et al. Metformin use is associated with reduced mortality in a diverse population with Covid-19 and diabetes. medRxiv [Preprint]. 2020. https://doi.org/10.1101/ 2020.07.29.20164020. Update in: Front Endocrinol (Lausanne). 2021;11:600439

Publisher's Note Springer Nature remains neutral with regard to jurisdictional claims in published maps and institutional affiliations. 